\title{
Synthetic Extracellular Matrices with Nonlinear Elasticity Regulate Cellular Organization
}

\author{
Kaizheng Liu, ${ }^{\dagger}$ Silvia M. Mihaila, ${ }^{\ddagger}, \$$ Alan Rowan, ${ }^{\S}$ Egbert Oosterwijk, ${ }^{\ddagger}$ and Paul H. J. Kouwer* ${ }^{\dagger} \uparrow$ \\ ${ }^{\dagger}$ Radboud University, Institute for Molecules and Materials, Heyendaalseweg 135, 6525 AJ Nijmegen, The Netherlands \\ ${ }^{*}$ Radboud University Medical Centre and Radboudumc Amalia Childern’s hospital, Radboud Institute for Molecular Life Sciences, \\ Department of Urology, Geert Grooteplein 26-28, PO Box 9101, 6500 HB Nijmegen, The Netherlands \\ ${ }^{\S}$ The University of Queensland, Australian Institute for Bioengineering and Nanotechnology, Brisbane, QLD 4072, Australia
}

\section{Supporting Information}

ABSTRACT: One of the promises of synthetic materials in cell culturing is that control over their molecular structures may ultimately be used to control their biological processes. Synthetic polymer hydrogels from polyisocyanides (PIC) are a new class of minimal synthetic biomaterials for threedimensional cell culturing. The macromolecular lengths and densities of biofunctional groups that decorate the polymer can be readily manipulated while preserving the intrinsic nonlinear mechanics, a feature commonly displayed by fibrous

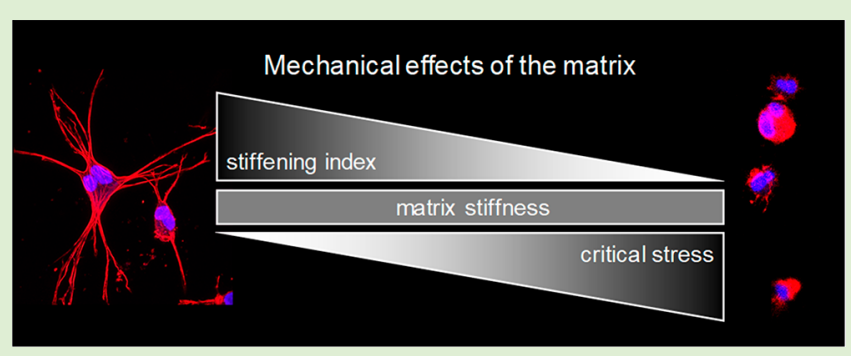
biological networks. In this work, we propose the use of PIC gels as cell culture platforms with decoupled mechanical inputs and biological cues. For this purpose, different types of cells were encapsulated in PIC gels of tailored compositions that systematically vary in adhesive peptide (GRGDS) density, polymer length, and concentration; with the last two parameters controlling the gel mechanics. Both cancer and smooth muscle cells grew into multicellular spheroids with proliferation rates that depend on the adhesive GRGDS density, regardless of the polymer length, suggesting that for these cells, the biological input prevails over the mechanical cues. In contrast, human adipose-derived stem cells do not form spheroids but rather spread out. We find that the morphological changes strongly depend on the adhesive ligand density and the network mechanics; gels with the highest GRGDS densities and the strongest stiffening response to stress show the strongest spreading. Our results highlight the role of the nonlinear mechanics of the extracellular matrix and its synthetic mimics in the regulation of cell functions.

\section{INTRODUCTION}

An increasing number of reports reveal that the mechanical properties of the extracellular matrix (ECM) play a crucial role in the regulation of cell function. ${ }^{1,2}$ Adherent cells attach to the matrix via integrin-matrix protein binding and sense ECM mechanics with the aid of a large number of adhesionassociated proteins and mechanotransduction pathways. ${ }^{1}$ These physical signals synergize with the chemical signals and simultaneously and coherently orchestrate cell fate, including cellular organization, proliferation, migration, (stem cell) differentiation, and self-renewal. ${ }^{2,3}$ It should be noted that in vivo, cells reside in a soft three-dimensional (3D) microenvironment and it has become increasingly clear that (stiffer) 2D substrates are insufficiently capable to simulate the complex processes that occur in $3 \mathrm{D}$ and thus are not representative of a realistic situation. When 3D cell-matrix adhesion interactions are taken as an example, they differ from their $2 \mathrm{D}$ counterpart in the content of $\alpha_{5} \beta_{1}$ and $\alpha_{\mathrm{v}} \beta_{3}$ integrins, paxillin, tyrosine phosphorylation of focal adhesion kinase (FAK), and other cytoskeleton proteins. ${ }^{4}$ Hence, to replicate the in vivo-like settings, the establishment of accurate $3 \mathrm{D}$ culture models has become crucial. ${ }^{4,5}$
The natural ECM is a sophisticated system that is composed of numerous elements that together provide the right chemical, biological, and mechanical environment for cells. From an architectural point of view, the fibrous components of the ECM play a main role in maintaining the structural integrity of the system and, as such, contribute to the bulk mechanical properties. $^{6}$ As a result of their fibrous nature and high persistence lengths, these biopolymers are able to form stiff networks at very low concentrations, with large pores that allow for diffusion of large molecules as well as cell migration. In addition, these biopolymer networks typically possess intriguing mechanical properties: they become many times stiffer when a small strain is applied. This effect, which is known as stress-stiffening or strain-stiffening, ${ }^{7}$ enables natural tissues to dynamically adjust the local mechanics in response to small cellular forces, generated when cells anchor to the network and physically pull it. Stress-stiffening is believed to play a role in the prevention of tissue rupture, transduction of cellular forces, ${ }^{8}$ enabling of contractile cells to communicate

Received: September 28, 2018

Revised: December 28, 2018

Published: January 4, 2019 


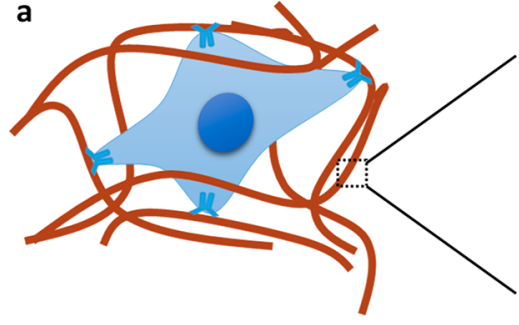

semi-flexible b polymer network

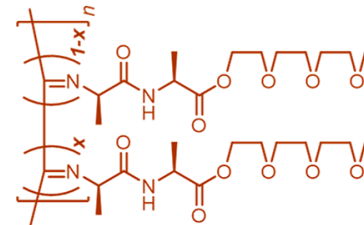

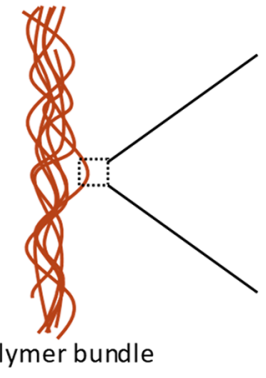

polymer bundle

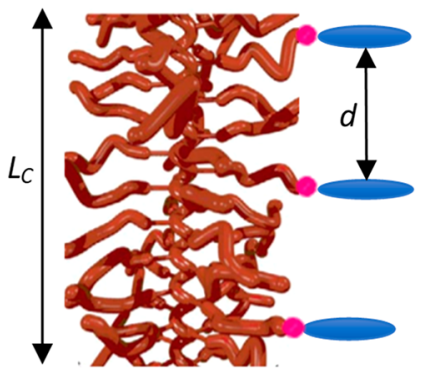

single chain with peptides

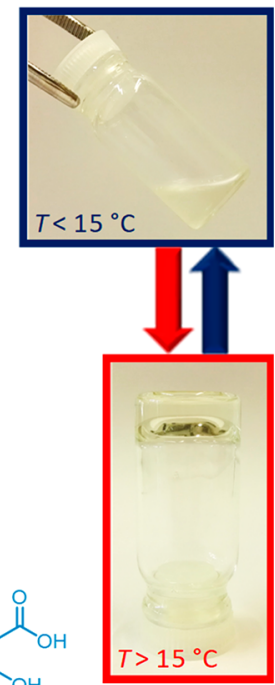

Figure 1. Structure and hierarchical assembly of the polyisocyanide (PIC)-based gels as a reductionist model for the ECM. (a) The semiflexible network is composed of bundles of polymer chains with a broad pore size distribution ranging from nanometers to microns. The polymer contour length, $L_{\mathrm{C}}$, and the density of peptide adhesion sites, given by the average distance $d$ between two adjacent groups, was varied in this work. (b) The molecular structure of the polymer is shown in red, the GRGDS peptide is shown in blue, and the linking group is shown in pink, matching with the colors in panel a. (c) The PIC polymers reversibly gel above the gelation temperature $T_{\text {gel }} \sim 20^{\circ} \mathrm{C}$.

their local position, ${ }^{9}$ and in guiding stem cell differentiation. ${ }^{10}$ Note that this effect is not to be confused with stiffening through the irreversible (strain-induced) formation of additional cross-links in the gel. ${ }^{11,12}$ Recently, there have been an increasing number of reports identifying the possible roles of a dynamic microenvironment as well as the reproduction of the dynamics of natural tissues. ${ }^{13}$ In fact, many reconstituted gels of ECM proteins (collagen, fibrin, etc.) exhibit some degree of stress relaxation, ${ }^{14}$ which reduces the stiffness of the matrix in time when the material is strained. Through this mechanism, cellular traction forces relax, which allows for matrix remodeling. Although different groups are studying these phenomena separately in the field of biomechanics, it is important to underline that nonlinear mechanics and viscoelasticity exist and function simultaneously in the dynamic remodeling of the matrix, ${ }^{15}$ albeit at different time scales.

Current 3D cell culture studies employ a large number of natural and semisynthetic polymer hydrogels based on, for instance, collagen, fibrin, hyaluronic acid, elastin, alginate, chitosan, etc. ${ }^{16-18}$ Some of these materials possess nonlinear mechanical properties; however, it is extremely difficult to control their properties precisely. For most of these materials, the characteristic approach for the manipulation of the (non)linear mechanics is to change the concentration of the polymers, which simultaneously impacts other features of the microenvironment such as the density of biological ligands, pore size, and porosity to name a few. ${ }^{18}$ Although many synthetic polymer hydrogels have been developed and utilized intensively for biomechanics studies, ${ }^{19,20}$ their architecture and linear mechanical properties make them often inadequate mimics to understand in vivo cell-matrix interactions. Despite the tremendous advances in the design of synthetic matrices, there is still an urgent need for synthetic polymer hydrogels that permit the independent decoupling of biochemical properties from the biomimetic mechanical properties. Such systems would enable the development of in vitro microenvironments that can be truly tailored to any biomedical application.
Recently, we introduced polyiscocyanide (PIC)-based hydrogels that are fully synthetic but strongly biomimetic in architecture and mechanics. ${ }^{21}$ On deformation beyond a critical stress, $\sigma_{\mathcal{c}}$ PIC gels can stiffen up a factor of 100 , analogous to fibrin and collagen. The key mechanical parameters in PIC gels, i.e., $\sigma_{\mathrm{c}}$ and the stiffening index $m$, metrics for the sensitivity and the responsiveness toward stress, respectively, indeed are similar to those found in these naturederived gels. More importantly, in PIC gels, these parameters are readily controlled by the molecular structure, the concentration and external conditions, such as temperature and salt concentrations. ${ }^{22-26}$ Moreover, as a thermoresponsive polymer, PIC chains bundle up and form interconnected fibers above the gelation temperature (typically $T_{\text {gel }} \sim 20{ }^{\circ} \mathrm{C}$ ) and the gels disassemble back to polymer solutions below $T_{\text {gel }}$, which enables researchers to easily encapsulate and harvest their cells. Earlier work has explored the use of PIC hydrogels as a guide for stem cell differentiation in $2 \mathrm{D}^{27}$ and $3 \mathrm{D}^{10}$ cultures, to support prevascularization, and as organotypic culturing support. $^{28}$

In this manuscript, we study how changes in the structure of the PIC polymer, i.e., the contour length $L_{C}$ and the adhesive peptide density $D$ on the polymer chain impact cellular behavior. For this study, we prepared a series of PIC polymers of different molecular weights and equipped them with adhesive peptides based on GRGDS (Figure 1a,b). We hypothesize that upon integrin-mediated binding of cells to the adhesive peptides on the polymer, the cells are able to generate internal stresses within the network and, as a result of the stress-sensitivity of PIC hydrogels, they are able to change the local mechanical microenvironment. By increasing/ decreasing $L_{\mathrm{C}}$, we change the gel stiffness, but more importantly, the sensitivity to stress without changing its concentration. ${ }^{22}$ Through the peptide density, we tune the number of cell-polymer contacts and with that, the efficiency of stress transfer from the cell to its microenvironment. In our study, we first probed different cell lines. As the most stringent 
effects were noticed with adipose-derived stem cells, we subsequently studied those more in-depth.

\section{EXPERIMENTAL SECTION}

Synthesis of Polyisocyanides. Polyisocyanides were synthesized as previously reported. ${ }^{29}$ In short, the isocyanide monomer was dissolved in freshly distilled toluene and stirred. Two parameters were adjusted: (i) the ratio of azide-functionalized and total monomer and (ii) the ratio $\mathrm{Ni}^{2+}$ to total monomer. All ratios are given in Table 1 .

\section{Table 1. Polymers and Characterization}

$\begin{array}{ccccc}\text { polymer } & {\left[\mathrm{Ni}^{2+}\right]:[\mathrm{M}] \mathrm{ratio}^{a}} & M_{\mathrm{v}}{ }^{b}\left(\mathrm{~kg} \mathrm{~mol}^{-1}\right) & L_{\mathrm{C}}{ }^{c}(\mathrm{~nm}) & \langle d\rangle^{d}(\mathrm{~nm}) \\ \text { PIC1 } & 1: 5000 & 562 & 223 & 3 \\ \text { PIC2 } & 1: 5000 & 575 & 228 & 10 \\ \text { PIC3 } & 1: 5000 & 558 & 221 & 25 \\ \text { PIC4 } & 1: 5000 & 539 & 214 & 50 \\ \text { PIC5 } & 1: 5000 & 568 & 225 & 100 \\ \text { PIC6 } & 1: 5000 & 568 & 225 & e \\ \text { PIC7 } & 1: 1000 & 263 & 106 & 10 \\ \text { PIC2 } & 1: 5000 & 575 & 228 & 10 \\ \text { PIC8 } & 1: 7000 & 589 & 236 & 10\end{array}$

${ }^{a}$ Catalyst:monomer ratio for the polymerization reaction. ${ }^{b} M_{\mathrm{v}}=$ Viscosity-based molecular weight of azide-appended polymers. ${ }^{c}$ Average contour length based on $M_{\mathrm{v}} \cdot{ }^{d}$ Average distance between peptides based on feed ratio in the polymerization. ${ }^{e}$ No GRGDS present in PIC6.

The appropriate amounts of monomers and catalyst solution $\mathrm{Ni}\left(\mathrm{ClO}_{4}\right)_{2} \cdot 6 \mathrm{H}_{2} \mathrm{O}\left(0.1 \mathrm{mg} \mathrm{ml}^{-1}\right.$ in freshly distilled toluene/absolute ethanol 9:1) were dissolved in toluene, and the final isocyanide concentration was adjusted to $50 \mathrm{mg} \mathrm{mL}^{-1}$. The mixture was stirred at room temperature, and the progress of the reaction was followed by IR-ATR (disappearance of the characteristic isocyanide absorption at $2140 \mathrm{~cm}^{-1}$ ). Once the polymerization was complete, the polymer was precipitated in diisopropyl ether under vigorous stirring and collected by centrifugation. The polymer was dissolved in dichloromethane, precipitated for another two rounds, and air-dried to yield the polymers as off-white solids. The molecular weight of the polymer was determined by viscometry (dilute solutions in acetonitrile) using the empirical Mark-Houwink equation $[\eta]=K M_{\mathrm{v}}{ }^{a}$, where $[\eta]$ is the experimentally determined intrinsic viscosity, $M \mathrm{v}$ is the viscositydetermined molecular weight, and the Mark-Houwink constants $K$ and $a$ depend on polymer characteristics and solvent, temperature, etc. We use parameters previously determined ${ }^{30}$ for other polyisocyanides: $K=1.4 \times 10^{-9}$ and $a=1.75$. The results are given in Table 1.

Conjugation of Adhesive Peptides. The peptide GRGDS was first conjugated to the $\mathrm{BCN}$-containing spacer following earlier described protocols. ${ }^{10}$ The peptide (H-Gly-Arg-Gly-Asp-Ser-OH, Bachem, Germany) was dissolved in borate buffer $(\mathrm{pH}=8.4)$ at 6 $\mathrm{mg} \mathrm{mL}^{-1}$. The dissolved BCN-NHS linker (Synaffix, The Netherlands) in DMSO $\left(6 \mathrm{mg} \mathrm{mL}^{-1}\right)$ was added to the peptide solution in borate buffer in a 1.1:1 molar ratio and stirred on the stirring plate for $24 \mathrm{~h}$ at room temperature. The formation of the BCN-GRGDS conjugate was confirmed by mass spectrometry $\left(M=911.4 \mathrm{~g} \mathrm{~mol}^{-1}\right)$. The azide-appended polymer (PIC1-PIC5, PIC7, PIC8) was dissolved in acetonitrile $\left(2.5 \mathrm{mg} \mathrm{mL}^{-1}\right)$, and the appropriate volume of the BCN-GRGDS solution in borate buffer (95\% molar equivalent of the corresponding azide amount on the polymer) was added. The solution was stirred for $24 \mathrm{~h}$ at room temperature. The polymerpeptide conjugates were precipitated in diisopropyl ether, collected by centrifugation, and air-dried for $24 \mathrm{~h}$.

Rheological Analysis of Polymer Hydrogels. For the mechanical analysis of the gels, a stress-controlled rheometer (Discovery HR-1 or HR-2, TA Instruments) with an aluminum or steel parallel plate geometry was used (diameter $=40 \mathrm{~mm}$, gap $=$ $500 \mu \mathrm{m}$ ). All samples were loaded onto the rheometer plate in the liquid state at $T=5{ }^{\circ} \mathrm{C}$ followed by a temperature ramp to $T=37^{\circ} \mathrm{C}$ at a rate of $1.0^{\circ} \mathrm{C} \mathrm{min}^{-1}$. The moduli were measured in the linear viscoelastic regime at amplitude of $\gamma=0.02$ or 0.04 and a frequency of $\omega=1.0 \mathrm{~Hz}$. The sample was allowed to equilibrate at $37^{\circ} \mathrm{C}$ prior to the nonlinear measurements. Here, the gel was subjected to a constant prestress of $\sigma_{0}=0.5$ to $200 \mathrm{~Pa}$, and the differential modulus $K^{\prime}$ was probed with a small superposed oscillatory stress at frequencies of $\omega=$ 10 to $0.1 \mathrm{~Hz}$ (reported data at $\omega=1 \mathrm{~Hz}$ ). The oscillatory stress was at least 10 times smaller than the applied prestress.

Cell Culture and Encapsulation. Human bladder smooth muscle cells were purchased from ScienCell and cultured in smooth muscle cell medium with growth supplement (ScienCell, USA). T24 and Hela cells were purchased from ATCC; SKRC52 cells were obtained from a mediastinal metastasis of a primary RCC2. All cancer cells were cultured in RPMI 1640 with GlutaMax (Gibco, Thermo Fisher, USA). Human adipose-derived stem cells were obtained from the Radboud Biobank and cultured in minimum essential medium eagle $(\alpha$-MEM) (Invitrogen, Thermo Fisher, USA). All media were supplemented with $10 \%$ fetal bovine serum (Sigma-Aldrich, USA) and $1 \%$ penicillin/streptomycin (final concentration of $100 \mathrm{IU} / \mathrm{mL}$ penicillin and $100 \mu \mathrm{g} / \mathrm{mL}$ streptomycin, Gibco, Thermo Fisher, USA).

Dry PIC polymers were sterilized by UV for $20 \mathrm{~min}$ and then dissolved in medium for $24 \mathrm{~h}$ at $4{ }^{\circ} \mathrm{C}$. Cells were harvested by trypsin treatment once they reached $100 \%$ confluence and were resuspended in fresh medium. Cell densities were determined by a LUNA-FL dual fluorescence cell counter. Cells were mixed with the polymer solution on ice with a predetermined ratio to achieve the required cell density and polymer concentration. After mixing, the solutions were transferred to 48-well plates (Corning, USA) or 8-well chambered cover slides (Sigma-Aldrich, USA) and heated to $37{ }^{\circ} \mathrm{C}$ where gelation occurred. After gel formation, culture medium (at $37^{\circ} \mathrm{C}$ ) was added onto the samples. Then all samples were subject to standard cell culture conditions $\left(37{ }^{\circ} \mathrm{C}, 5 \% \mathrm{CO}_{2}\right)$. Note that no stem cell differentiation kit was added into the culture medium of the hACSs, and the cells are expected to maintain the stemness. ${ }^{10}$

Bright Field Imaging and Cell Morphology Analysis. Bright field images of cells encapsulated in hydrogels were acquired on a Leica DC200 microscope. Outlines of representative hASCs were plotted manually, and the quantitative analysis of circularity was performed by Fiji. A paired sample $t$ test was used to determine the statistical significance.

Cytoskeleton Staining and Confocal Microscope Imaging. Gels with encapsulated cells were washed with $0.9 \% \mathrm{NaCl}$ and then fixed with $3 \%$ paraformaldehyde in $0.9 \% \mathrm{NaCl}$ for $40 \mathrm{~min}$. After fixation, the samples were permeabilized with $0.1 \%$ Triton $\mathrm{X}-100$ in $0.9 \% \mathrm{NaCl}$ for $10 \mathrm{~min}$ and blocked with $1 \% \mathrm{BSA}$ in $0.9 \% \mathrm{NaCl}$ for 30 min. They were then incubated with Texas Red-X Phalloidin (50 IU/ $\mathrm{mL}$ in $1 \% \mathrm{BSA} / 0.9 \% \mathrm{NaCl}$, Sigma-Aldrich, USA) for $1 \mathrm{~h}$ and DAPI (5 $\mathrm{mg} \mathrm{mL}^{-1}$ in $0.9 \% \mathrm{NaCl}$, Thermo Fisher, USA) for $10 \mathrm{~min}$. All procedures above were performed at $37^{\circ} \mathrm{C}$. An Olympus FluoView 1000 confocal laser scanning microscope was used for fluorescence imaging. The temperature of the sample was kept at $37{ }^{\circ} \mathrm{C}$ by the heating element of the microscope.

Cell Proliferation Assay. (a) WST-1: Culture medium was gently removed, the new medium supplemented with the cell proliferation reagent WST-1 (Roche, Switzerland) at a final concentration of 1:10 (WST-1 stock solution/total working solution) was added, and the culture plates were incubated at $37{ }^{\circ} \mathrm{C}, 5 \% \mathrm{CO}_{2}$ for $2 \mathrm{~h}$. The absorbance was measured at $\lambda=450 \mathrm{~nm}$ with a plate reader (PerkinElmer 1420 Multilabel Counter). All samples were measured in triplicates. (b) Quant-iT PicoGreen dsDNA Assay Kit: The amount of dsDNA in each sample was quantified according to the kit manual (Invitrogen, Thermo Fisher, USA). In brief, $28.7 \mu \mathrm{L}$ of sample, 71.3 $\mu \mathrm{L}$ of $1 \mathrm{X}$ PicoGreen solution, and $100 \mu \mathrm{L} 1 \times \mathrm{TE}$ were mixed and incubated in a 96-well plate in the dark for $10 \mathrm{~min}$ and the fluorescence was read with a plate reader $\left(\lambda_{\text {excitation }}=485 \mathrm{~nm}, \lambda_{\text {emission }}\right.$ $=528 \mathrm{~nm}$ ). All samples were measured in triplicates. 


\section{RESULTS AND DISCUSSION}

Synthesis of the PIC-GRGDS Polymers and Gel Preparation. The generic polymer structure of the materials used in this work is shown in Figure 1b. For precursors, we prepared azide-appended PIC polymers PIC1-PIC8 by copolymerization of the $\mathrm{N}_{3}$-functionalized monomer with the nonfunctional monomer, following earlier reported procedures. ${ }^{10}$ The polymer contour length, $L_{C}$, was tuned by changing the total monomer to initiator ratio and calculated from the viscosity averaged molecular weight. ${ }^{22}$ Although at gel formation, the polymers bundle and form infinitely long networks, the length of the individual polymer chains is an important parameter in the linear and nonlinear mechanical properties of the gels. ${ }^{22}$ After purification of the polymers, the appropriate amounts of the GRGDS peptide, previously equipped with a $\mathrm{BCN}$ group, ${ }^{10}$ were added to the polymers, and the GRGDS-decorated polymers were collected after precipitation. As their concentration is very low, a quantitative characterization of the degree of peptide conjugation is challenging. On the basis of the high reaction rate of this conjugation chemistry ${ }^{31}$ and the results of earlier reports, ${ }^{29}$ we assume complete conversion and we present GRGDS spacings, $d$, based on the azide monomer fractions (Table 1, series polymer PIC1-PIC6). To vary $L_{C}$, we prepared a series of three polymers with the same spacing $(d=10 \mathrm{~nm})$ and different catalyst/monomer ratios (PIC7-PIC2-PIC8). Although the experimental values of the viscosity-averaged molecular weights, $M_{v}$, of PIC2 and PIC8 are very similar, we do find the expected differences in the mechanical properties of the corresponding gels.

For analysis and cell culture studies, the appropriate amount of the azide or GRGDS-functionalized polymers were dissolved in $\alpha$-MEM medium at $4{ }^{\circ} \mathrm{C}$ for $24 \mathrm{~h}$. Instantaneous and reversible gelation takes place upon heating above $T_{\text {gel }}$ (Figure 1c).

Mechanical Characterization. The PIC hydrogels were subjected to rheological analysis (Table 2). At low strain, we measure the storage modulus $G^{\prime}$ in the linear viscoelastic regime as a function of temperature (Figure S1). At high

Table 2. Hydrogel Samples for Cell Culture Experiments

$\begin{array}{cccccc}\text { hydrogel } & \text { polymer } & c^{a}\left(\mathrm{~g} \mathrm{~L}^{-1}\right) & D^{b}(\mu \mathrm{M}) & G^{\prime c}(\mathrm{~Pa}) & \sigma_{\mathrm{c}}^{c}(\mathrm{~Pa}) \\ \text { P1 } & \text { PIC1 } & 2.0 & 181 & 228 & 19.1 \\ \text { P2 } & \text { PIC2 } & 2.0 & 55 & 347 & 26.6 \\ \text { P3 } & \text { PIC3 } & 2.0 & 22 & 442 & 33.3 \\ \text { P4 } & \text { PIC4 } & 2.0 & 11 & 586 & 35.3 \\ \text { P5 } & \text { PIC5 } & 2.0 & 5 & 388 & 29.9 \\ \text { P6 } & \text { PIC6 } & 2.0 & d & 382 & 23.5 \\ \text { S1 } & \text { PIC7 } & 1.5 & 41 & 128 & 14.6 \\ \text { S2 } & \text { PIC2 } & 1.5 & 41 & 177 & 19.1 \\ \text { S3 } & \text { PIC8 } & 1.5 & 41 & 247 & 21.4 \\ \text { S4 } & \text { PIC7 } & 3.0 & 83 & 463 & 29.5 \\ \text { S5 } & \text { PIC2 } & 3.0 & 83 & 647 & 39.7 \\ \text { S6 } & \text { PIC8 } & 3.0 & 83 & 831 & 47.4 \\ \text { S7 } & \text { PIC7 } & 2.0 & 55 & 287 & 22.4 \\ \text { S8 } & \text { PIC8 } & 2.0 & 55 & 489 & 35.7\end{array}$

${ }^{a_{c}}=$ polymer concentration. ${ }^{b} D=$ density of conjugated GRGDS peptide. ${ }^{c}$ Shear modulus $\left(G^{\prime}\right)$ and critical stress $\left(\sigma_{\mathrm{c}}\right)$ measured at $T=$ $37{ }^{\circ} \mathrm{C}$ in $\alpha$-MEM medium. ${ }^{d}$ No GRGDS conjugated to PIC6. ${ }^{e} \mathbf{S} 7$ and S8 were not used for cell experiments but were included for a comprehensive view of the mechanical properties of the PIC gels. stresses, the gels reversibly stiffen and we describe the mechanical properties by the more relevant differential modulus $K^{\prime}=\partial \sigma / \partial \gamma$, where $\sigma$ and $\gamma$ are the stress and strain, respectively. Note that below a critical stress $\sigma_{\mathcal{c}}$ i.e., at $\sigma<\sigma_{\mathcal{c}}$ $K^{\prime}=G^{\prime}$, and at $\sigma>\sigma_{\mathcal{c}}, K^{\prime}$ depends on the applied stress $G^{\prime} ; G^{\prime}$ $\sim \sigma^{m}$, where $m$ is the stiffening index. The parameters $\sigma_{\mathrm{c}}$ and $m$ represent the sensitivity and responsiveness to macroscopic deformation.

Polymers PIC1-PIC6 have the same contour length $L_{\mathrm{C}}$ but different GRGDS loading $(d)$, and all form soft hydrogels with $G^{\prime}=230$ to $590 \mathrm{~Pa}$ at polymer concentration $c=2 \mathrm{mg} \mathrm{mL}^{-1}$ in medium at a physiological temperature of $T=37^{\circ} \mathrm{C}$ (Figure $2 a)$. Beyond the linear viscoelastic regime, the gels stiffen. Again at $2 \mathrm{mg} \mathrm{mL}^{-1}$, we find for P1-P6, a critical stress $\sigma_{\mathrm{c}}=$ 19 to $35 \mathrm{~Pa}$ and a stiffening index $m \approx 1$. As expected, for polymers with the same molecular weight, ${ }^{22}$ the curves collapse to a single master curve when the data is scaled to the storage modulus in the linear viscoelastic regime $G^{\prime}$ and $\sigma_{\mathrm{c}}$ (Figure $S 1)$. We do not find a clear correlation between the mechanical properties of the gel and the peptide loading (Table 2). Low peptide densities stiffen the gel slightly, but at the highest GRGDS densities, the gel softens. This nonlinear behavior results from a balance in effects introduced with the peptide. On one hand, the peptide makes the polymer slightly more hydrophilic, which increases $T_{\text {gel }}$ and thus decreases $G^{\prime}$ at $T=$ $37{ }^{\circ} \mathrm{C}$. On the other hand, the peptides add physical interactions between polymer chains, which will increase $G^{\prime}$. Overall, however, all gels are relatively soft, i.e., much softer than many other (synthetic) gels used for cell culture experiments, and display stress sensitivities that are biologically accessible. ${ }^{32}$ Gels $\mathbf{S 1 - S 6}$ of polymers with the same peptide density $D$ but of different lengths show an increase in both $G^{\prime}$ and $\sigma_{\mathrm{c}}$ with increasing $L_{\mathrm{C}}$ (Figure $2 \mathrm{~b}$ ). We used the gels at two different concentrations: $c=1.5$ and $3 \mathrm{mg} \mathrm{mL}^{-1}$, which gives gels with $G^{\prime}=100$ to $1000 \mathrm{~Pa}, \sigma_{\mathrm{c}}=14$ to $41 \mathrm{~Pa}$, and $D=41$ or $83 \mu \mathrm{M}$. The adhesive peptide concentrations in our gels are typically about an order of magnitude lower than in other commonly used synthetic gels. $14,33-35$

We find that softer gels are more stress-responsive (Figure $2 \mathrm{c})$, that is, they stiffen at lower stresses (low $\sigma_{\mathrm{c}}$ ). Interestingly, the stiffening index of gel series $\mathbf{S 1 - S 6 ~ n e g a t i v e l y ~ c o r r e l a t e s ~ t o ~}$ the critical stress (Figure 2d), which underlines that softer PIC hydrogels with a higher stress-sensitivity also possess a higher responsiveness (high $\mathrm{m}$ ). The fibrous architecture of the gels does not change significantly with the polymer chain length (in this studied regime $)^{24,36}$ which means that only a change in the architecture of the network is expected from the change in concentration, which for a concentration doubling gives a minor reduction of the mesh size $\xi$ as follows: $\xi \sim c^{-0.5}$.

Adhesive Peptide Density: Cell Proliferation vs Spreading. For cell culture studies, the cells were suspended in a cold $\left(0-5{ }^{\circ} \mathrm{C}\right)$ polymer solution in medium and immediately warmed to $37{ }^{\circ} \mathrm{C}$ to form $3 \mathrm{D}$ cell-gel constructs. Then the samples were cultured at standard conditions for 7 days. Bright field images show that the cell morphologies depend on the cell type and GRGDS concentration but much less on the gel properties (Figure 3a,b). Human bladder smooth muscle cells (hbSMCs), HeLa, SKRC52, and T24 cells tend to proliferate and organize into multicellular spheroids with diameters of tens of microns in all polymers (see Figure $3 \mathrm{a}$ and Figures S2-S5 for a full overview). The spatial distribution of cell nuclei and the arrangement of F-actin, however, differs for each cell type (Figure 3c). The 

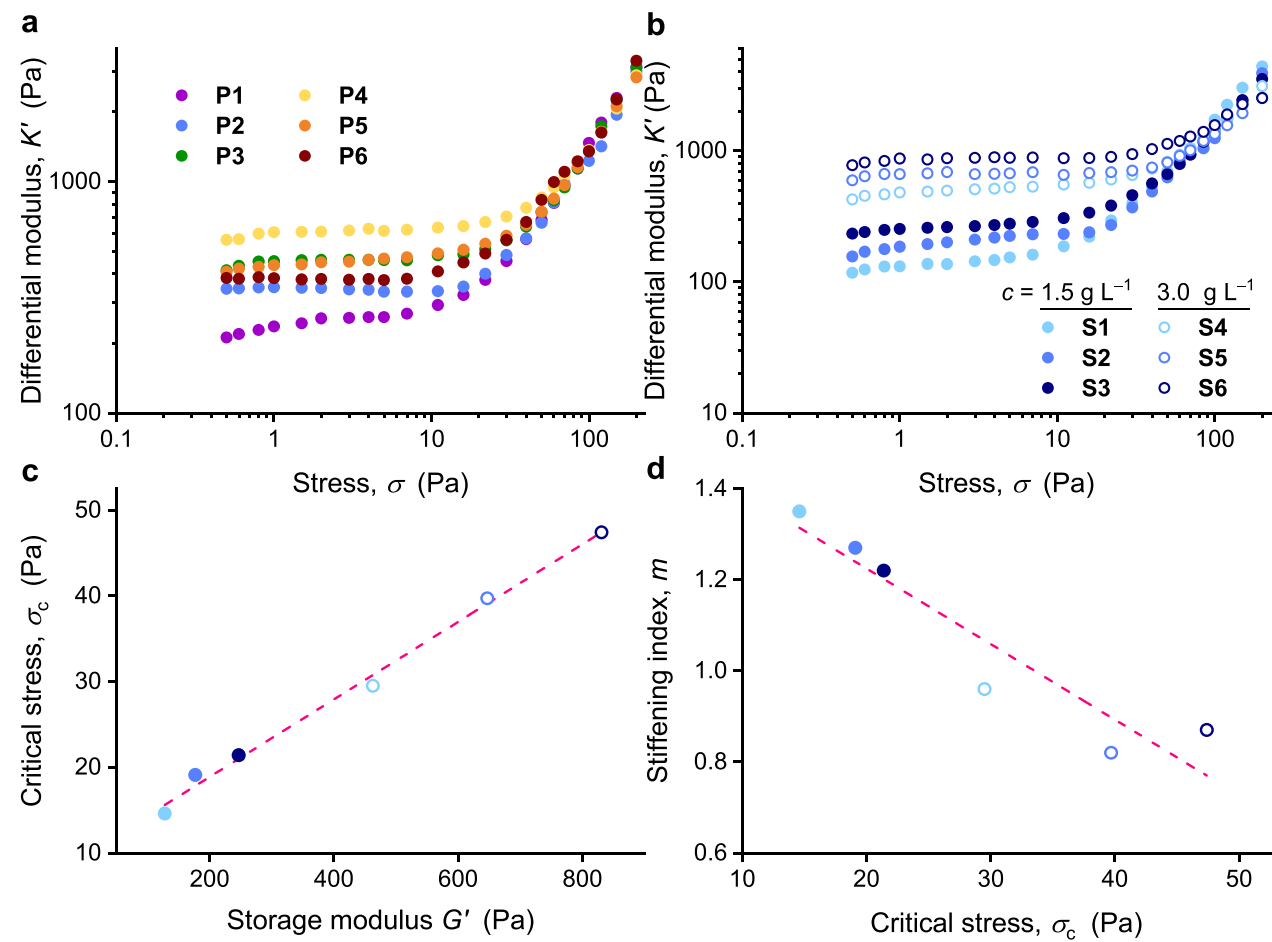

Figure 2. Mechanical properties of PIC gels used in this work. (a) Differential modulus $K^{\prime}$ as a function of applied stress $\left(c=2 \mathrm{mg} \mathrm{mL}^{-1}\right.$ in medium, $T=37^{\circ} \mathrm{C}$ ) for gels P1-P6. The plateau values at $\sigma<10$ Pa correspond to the modulus in the linear viscoelastic regime. (b) $K^{\prime}$ of gels S1-S6 (from PIC7, PIC2, and PIC8) with different length $L_{\mathrm{C}}$ but a fixed ligand density, at polymer concentrations $c=1.5 \mathrm{mg} \mathrm{mL}^{-1}$ (solid symbols) and $3.0 \mathrm{mg} \mathrm{mL}^{-1}$ (open symbols) in medium. (c,d) Linear correlations between the critical stress $\sigma_{\mathrm{c}}$ and the storage modulus $G^{\prime}$ and between the stiffening index $m$ and $\sigma_{\mathrm{c}}$ for S1-S6; $1.5 \mathrm{mg} \mathrm{mL}^{-1}$ (solid symbols) and $3.0 \mathrm{mg} \mathrm{mL}^{-1}$ (open symbols). The dashed line is a guide to the eye. The last two diagrams clearly show that the more stress-sensitive gels are both softer and more responsive toward applied stress. All measurements were done in $\alpha$-MEM medium at $37{ }^{\circ} \mathrm{C}$.

proliferation rates of these cell types are positively correlated to the density of adhesive ligands (Figure $3 \mathrm{~d}-\mathrm{g}$ ): gels with the highest GRGDS loading show highest proliferation rates. The relatively small difference in the mechanical properties in P1P6 has a minor effect on cell behavior. Human adipose-derived stem cells (hASCs) do not organize into large multicellular spheroids but rather show distinct morphological changes with peptide density. Cell protrusion and elongation are seen earlier (day one, see Figure S6) in gels with higher GRGDS densities (P1 and P2), compared to the other gels. After 7 days, the stem cells adopted a flattened morphology in $\mathbf{P 1}$ and P2, while only small protrusions developed in P3-P5, and no protrusions were observed in P6 (Figure 3b).

We observe that the number of stem cells decreases slightly in samples with low peptide densities, which we attribute to an insufficient adhesive ligand density. For the other four cell types, this was not the case; after 7 days, their cell number increased despite the lack of adhesive sites. We attribute this relatively efficient proliferation to the cell-cell interactions in the multicellular spheroids. In addition, the formation of multicellular structures is a very complex mechanism with rich biological content, where besides cell-matrix interactions, cell-cell contacts play a major role. ${ }^{37}$ In this work, however, we will not discuss this in further detail but restrict ourselves to the stem cells that seem most sensitive to the RGD concentrations of the synthetic biomimetic hydrogels.

We studied cell spreading as an indicator for a plethora of cell functions. ${ }^{38}$ Although commonly used in $2 \mathrm{D}$ cell studies, spreading studies seem less popular in 3D culture studies. Earlier work that actually focused on cell spreading on planar surfaces proved the existence of a critical adhesive ligand density (e.g., $70 \mathrm{~nm}$ for mesenchymal stem cells on nanopatterned PEG matrix) for efficient cell spreading and focal adhesion formation. ${ }^{39}$ 3D cell-matrix adhesions, however, are distinct from focal adhesions that are found on two-dimensional planar surfaces. ${ }^{4}$ In this work, we focus on cell spreading in a $3 \mathrm{D}$ fibrous network with a controlled ligand density.

When polymer length and polymer concentration are fixed, our results reveal that microenvironments rich in adhesion sites promote stem cell spreading and the formation of cellular networks by providing more molecular anchoring points for integrins on the cell membrane. In other words, it is easier for cells to anchor to the artificial ECM and initiate actin polymerization, myosin contraction, and adhesion protein recruitment. ${ }^{40}$ In our case, the minimum required adhesive peptide density for hASCs to spread is $\sim 55 \mu \mathrm{M}$ (Figure $3 \mathrm{~b}$ ). This density corresponds to a $\sim 10 \mathrm{~nm}$ distance between adjacent peptides on a polymer chain, a distance that is of the same order of magnitude as the length of the $\alpha_{5} \beta_{1}$ integrin head $^{41}$ (that is overexpressed in human mesenchymal stem cells). When two adjacent peptides are too far apart, hASCs seem unable to generate enough traction to modulate their morphology. Once more, we iterate that the RGD density used in our study is low compared to the RGD content inside other synthetic cell-laden hydrogels. ${ }^{14,33-35}$ We propose that the bundling of PIC polymers results in the clustering of RGD peptides, which results in more effective cell spreading. ${ }^{42}$ Notably, we also encapsulated hASCs in P1-P6 with a higher 

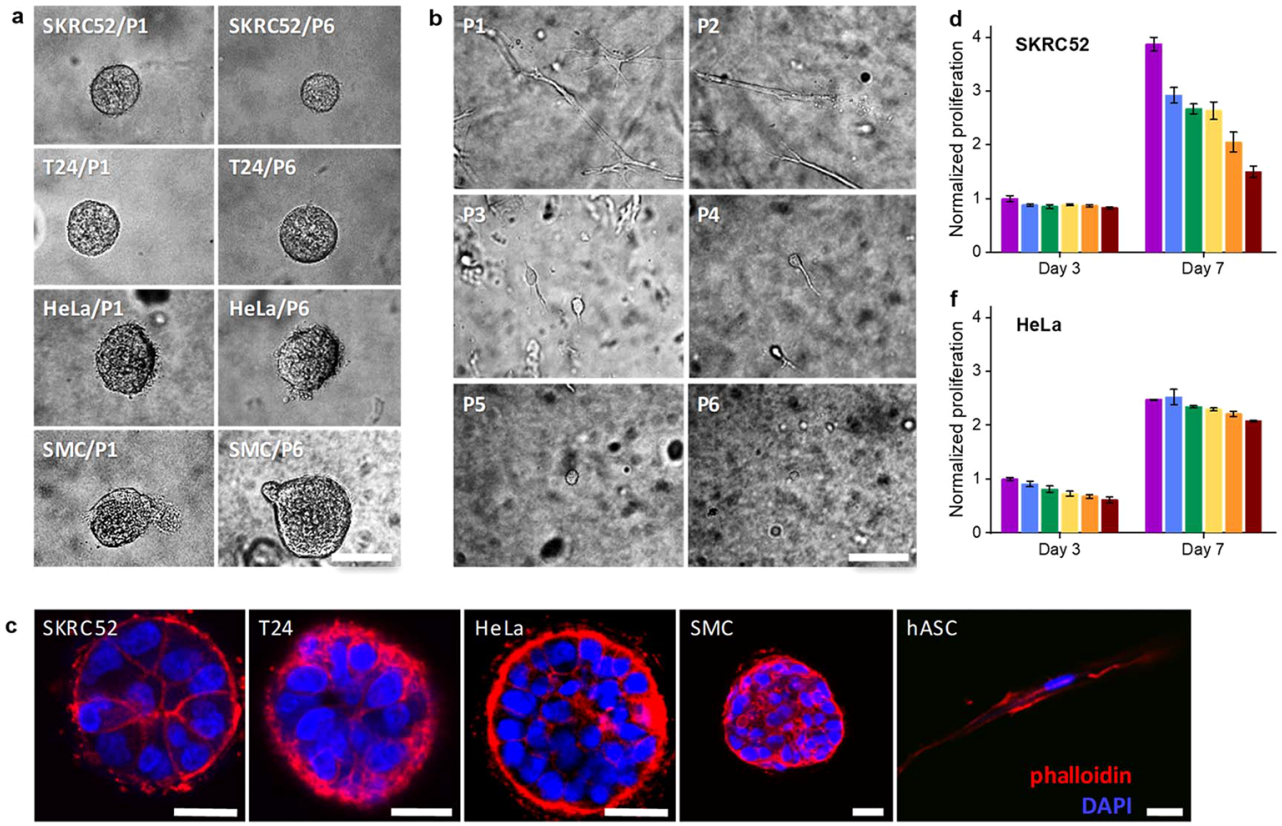
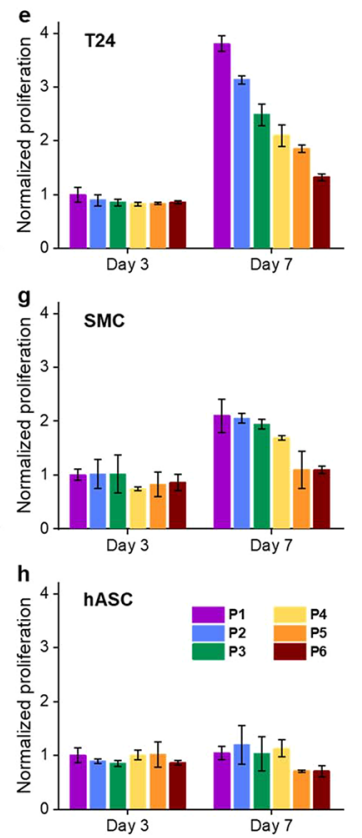

Figure 3. Cell behavior in PIC gels with different GRGDS densities based on P1-P6. (a) SKRC52s, T24s, HeLas, and SMCs in P1 and P6 after 7 days of culturing. Irrespective of the peptide density, cell spheroids are formed for all cell types. (b) hACSs show strongly different morphologies in P1-P6 after 7 days of culturing: elongated morphologies at the highest GRGDS densities and spherical morphologies at low densities. (c) Fluorescence staining with Phalloidin (red, F-actin) and DAPI (blue, cell nuclei) of different cell types after 7 days of culturing in P2 show that the multicellular aggregate differs in nuclear and cytoskeletal arrangement. Contrarily, hACSs show single elongated morphologies. ( $\mathrm{d}-\mathrm{h})$ Proliferation, normalized to absorbance of $\mathbf{P 1}$ at day 3, for different cell types after 3 and 7 days of culturing in P1-P6. SKRC52s, T24s, HeLas, and SMCs show increased proliferation with higher peptide densities. For hASCs, proliferation in all gels is low. Note that the colors in $\mathrm{d}-\mathrm{h}$ match those in Figure 2a. For all samples, the PIC concentration $c=2 \mathrm{mg} \mathrm{mL}^{-1}$ and cell density is 20000 cells mL $\mathrm{mL}^{-1}$. The scale bar for all figures is $70 \mu \mathrm{m}$. The error bars in $\mathrm{d}-\mathrm{h}$ represent standard deviations of three experiments.

cell density ( 200000 cells $\left.\mathrm{mL}^{-1}\right)$ and found a similar trend in morphology and proliferation (Figures S8 and S9).

Nonlinear Mechanics of the ECM Regulates the Organization of hASCs. Nonlinear mechanics is frequently observed in gels of natural polymers, but to this date, many biological effects of stress-stiffening and their mechanisms still remain to be unravelled. ${ }^{1,10}$ We prepared six gels S1-S6 with different critical stresses and thus different responses to stress that is applied externally (rheometer) or internally (cells) to screen the influence of ECM stress-stiffening on cell spreading of 3D hASC cultures. The PIC polymers were conjugated with a fixed density of adhesive peptides with $\langle d\rangle=10 \mathrm{~nm}$ to provide the essential amount of attachment sites. After culturing for 3 days, the cells encapsulated in S1-S6 were analyzed (Figure 4) using bright field microscopy (Figure 4a) and after staining the nuclei and F-actin, the cells were analyzed using confocal microscopy (Figure $4 \mathrm{~b}$ ). The extent of spreading of the cells was quantified by plotting and measuring the circularity of ten representative cells (Figure 4c,d). At low gel concentrations $\left(c=1.5 \mathrm{mg} \mathrm{mL}^{-1}\right)$, the stem cells show significantly better spreading (smaller circularity) in samples with a smaller critical stress (series $\mathbf{S 1 - S 3}$ ). At $3 \mathrm{mg} \mathrm{mL}^{-1}$ (S4-S6), a plateau is reached (Figure 4e). We did not detect any significant proliferation until day 3 , which is in line with the results from P1-P6 (Figure 4f).

A wide variety of cellular processes, including sensing of ECM mechanics, cell-cell communication, on/off switching of functional binding sites, and alteration of enzymatic ECM degradation, ${ }^{43}$ involve cell anchoring to the ECM fibers and the straining of the fibers that induces a stiffening response in the matrix. The storage modulus (i.e., the stiffness in the linear viscoelastic regime) of the PIC gels falls into the category of soft biomaterials, ${ }^{18}$ which means that primarily the difference in critical stress governs the real mechanical properties of the microenvironment of the encapsulated cells. A lower critical stress implies that the material is more sensitive to cell-induced matrix stiffening. For gels that display large stiffening indices, i.e., gels that are more responsive toward contractile stresses applied by the cell, this effect is further enhanced. In these fibrous gels, cells are able to adhere and pull the PIC fibers, stiffen them, and accumulate enough traction force for the observed morphological changes. The culturing in gels that have a higher $\sigma_{\mathrm{c}}$ and a lower $m$ leads to the opposite effect. These materials remain relatively static; i.e., the cells are able to anchor due to the sufficient amount of adhesive peptides present but are unable to generate sufficient traction force to spread. Therefore, cells tend to maintain the rounded morphology and do not spread out. Despite the difference in initial low-stress plateau moduli, the role of nonlinear mechanics at higher stress is more dominant as these soft gels can stiffen up to kilopascals with external stress (Figure 2b).

In addition to the effects originating from changes in the macroscopic (nonlinear) mechanical properties, we observed cell alignment in some experiments. Despite the low cell density in the samples (down to 20000 hASCs $\mathrm{mL}^{-1}$ ), we found that in some of our gels, cells aligned and protrusions form in a common direction (Figure 4 a panel S1). The alignment implicates that isolated cells are able to induce longrange interactions by matrix remodeling and/or that fiber alignment occurs in the semiflexible polymer network, which allows neighboring cells to sense the fiber orientation and 


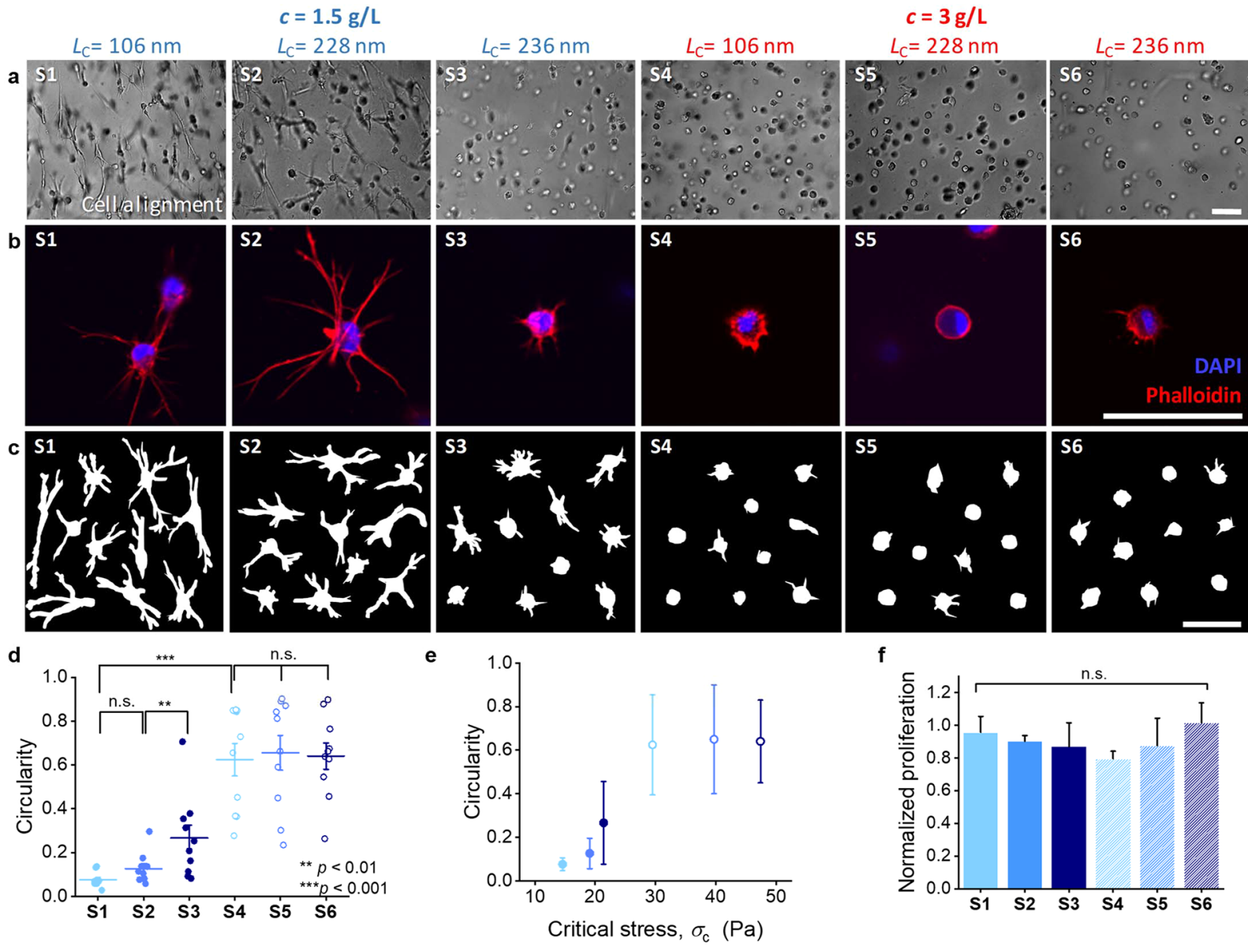

Figure 4. Influence of nonlinear mechanics of the PIC matrix on the spreading of hASCs. (a) Representative bright field images of hASCs. (b) Representative fluorescence images of hASCs; nuclei are stained with DAPI (in blue), and F-actin is stained using Texas Red Phalloidin (in red). (c) Cell outlines of ten representative cells. All images were taken 3 days after cell encapsulation; cell density $=200000$ cells $\mathrm{mL}^{-1}$, scale bars $=70$ $\mu \mathrm{m}$. (d) Quantified circularity of the cells in panel c for gel S1-S6. (e) Averaged circularity as a function of the corresponding critical stress $\sigma_{\mathrm{c}}$ of the gels. (f) Quantified cell proliferation using a PicoGreen assay, normalized by day 0 . Note that the colors in $\mathrm{d}-\mathrm{f}$ match those in Figure $2 \mathrm{~b}-\mathrm{d}$. For each sample, 10 cells were analyzed. Statistics: n.s. = not significant $(p>0.05),{ }^{*} p<0.01, * * * p<0.001$.

align. Similar findings have been observed and discussed earlier by several research groups. 8,44 These observations are in agreement with cell studies in collagen and fibrin. We note that fiber alignment will enhance (local) stress-stiffening behavior in PIC gels ${ }^{45}$ and subsequently influence cellular behavior. In addition, we point out that, over time, stem cells will secrete natural ECM that will contribute to the mechanical microenvironment of the cell.

\section{CONCLUSIONS}

It is universally acknowledged that the storage modulus is a key component of hydrogel mechanics that affect cellular behavior in $3 \mathrm{D}$ cell cultures. More recently, stress relaxation was identified as another important contributor. We wish to add to this perspective that nonlinear mechanics also strongly contribute to the mechanical spectra that cells are able to sense. In this work, we provide insight into the correlations between cellular biophysics and stress-stiffening of biomimetic polymer gels. In the presence of cells that apply contraction forces to the gel, the stiffness no longer remains a "static" constant but becomes a dynamic parameter that responds to cell-gel interactions, which can be tuned by the design of the polymer. In biological or biomimetic, fibrillar, and strain- stiffening matrices, it is insufficient to describe the mechanical properties of the gel by its "static" stiffness alone.

For cell culturing in synthetic matrices, which offer the advantage of tunable mechanical properties, one needs to engineer cell attachment sites. The density and distribution of adhesive peptides will play yet another important role in the accomplishment of desired cell behavior. Finally, different cells, cultured in the same matrix, will also respond differently. Our results once more underline that all these factors should be included in a comprehensive experimental design, but at the same time, it is still difficult to predict which of the parameters will dominate behavior and therefore be the most urgent to optimize.

Our minimal PIC hydrogel model is particularly suitable as a guide to further design soft biomimetic materials. As the next step of research, mechanical analyses of both bulk tissue constructs and the local niche is needed, for instance, it would be of great interest to characterize the (variation in) local matrix stiffness using microrheology. Ultimately, the comprehensive understanding of interactions at the cell-matrix interface should be utilized to benefit regenerative medicine and tissue engineering. 


\section{ASSOCIATED CONTENT}

\section{S Supporting Information}

The Supporting Information is available free of charge on the ACS Publications website at DOI: 10.1021/acs.biomac.8b01445.

Diagrams S1-S9, showing the mechanical properties of gels of P1-P6 (Figure S1); bright field cell culture images at day 1,3 , and 7 in gels of P1-P6 $(2.0 \mathrm{mg}$ $\mathrm{mL}^{-1}$ ) with hbSMCs (Figure S2); HeLas (Figure S3); T24s (Figure S4); SKRC52s (Figure S5) and hASCs (Figure S6); bright field cell culture images at day 0, 1, and 2 of hASCs in gels $\mathbf{S 1 - S 6}$ at $c=1.5$ and $3.0 \mathrm{mg}$ $\mathrm{mL}^{-1}$ (Figure S7); bright field cell culture images at day 1,3 , and 7 of hASCs in gels of P1-P6 at $c=2.0 \mathrm{mg}$ $\mathrm{mL}^{-1}$ at higher cell density 20000 cells $\mathrm{mL}^{-1}$ (Figure S8); crude WST-essay results (Figure S9); LIVE/DEAD staining of hASCs at $48 \mathrm{~h}$ in $\mathbf{P 2}$ (Figure S10) (PDF)

\section{AUTHOR INFORMATION}

\section{Corresponding Author}

*E-mail: p.kouwer@science.ru.nl. Phone: +31 243656424.

\section{ORCID}

Paul H. J. Kouwer: 0000-0002-2760-191X

\section{Present Address}

${ }^{\$}$ University Medical Center Utrecht, Department of Nephrology and Hypertension, Heidelberglaan 100, 3584 CX, Utrecht, Netherlands.

\section{Author Contributions}

The manuscript was written through contributions of all authors. All authors have approved the final version of the manuscript.

\section{Funding}

This project has received funding from the European Union's Horizon 2020 research and innovation program under the Marie Skłodowska-Curie grant agreements numbers 642687 (KL) and 626934 (SMM).

\section{Notes}

The authors declare no competing financial interest.

\section{ACKNOWLEDGMENTS}

We thank Dr. Susana Rocha for the valuable discussions during writing this manuscript, Huib Croes and Dr. Elisabeth Pierson for their technical assistance with confocal imaging, Onno van den Boomen for his support on polymer synthesis, Dorien Tiemessen for her support on the cell culture studies, Peter Heinz Janke for his support on the biological assays, and Dr. Jeannette Wakka-Oosterwijk and Kas Pleysier for their support on the cancer cell culture studies. This project has received funding from the European Union's Horizon 2020 research and innovation programme under the Marie Skłodowska-Curie grant agreements No 642687 (KL) and No 626934 (SMM).

\section{REFERENCES}

(1) Humphrey, J. D.; Dufresne, E. R.; Schwartz, M. A. Mechanotransduction and extracellular matrix homeostasis. Nat. Rev. Mol. Cell Biol. 2014, 15, 802-812.

(2) Vining, K. H.; Mooney, D. J. Mechanical forces direct stem cell behaviour in development and regeneration. Nat. Rev. Mol. Cell Biol. 2017, 18, 728.

(3) Scadden, D. T. The stem-cell niche as an entity of action. Nature 2006, 441, 1075-1079.
(4) Cukierman, E.; Pankov, R.; Stevens, D. R.; Yamada, K. M. Taking cell-matrix adhesions to the third dimension. Science 2001, 294, 1708-1712.

(5) Justice, B. A.; Badr, N. A.; Felder, R. A. 3D cell culture opens new dimensions in cell-based assays. Drug Discovery Today 2009, 14, 102-107.

(6) Tibbitt, M. W.; Anseth, K. S. Hydrogels as extracellular matrix mimics for 3D cell culture. Biotechnol. Bioeng. 2009, 103, 655-663.

(7) Storm, C.; Pastore, J. J.; MacKintosh, F. C.; Lubensky, T. C.; Janmey, P. A. Nonlinear elasticity in biological gels. Nature 2005, 435, 191-194.

(8) Rudnicki, M. S.; Cirka, H. A.; Aghvami, M.; Sander, Edward A.; Wen, Q.; Billiar, Kristen L. Nonlinear strain stiffening is not sufficient to explain how far cells can feel on fibrous protein gels. Biophys. J. 2013, 105, 11-20.

(9) Winer, J. P.; Oake, S.; Janmey, P. A. Non-linear elasticity of extracellular matrices enables contractile cells to communicate local position and orientation. PLoS One 2009, 4, e6382.

(10) Das, R. K.; Gocheva, V.; Hammink, R.; Zouani, O. F.; Rowan, A. E. Stress-stiffening-mediated stem-cell commitment switch in soft responsive hydrogels. Nat. Mater. 2016, 15, 318-325.

(11) Tibbitt, M. W.; Rodell, C. B.; Burdick, J. A.; Anseth, K. S. Progress in material design for biomedical applications. Proc. Natl. Acad. Sci. U. S. A. 2015, 112, 14444-14451.

(12) Tran, Y. H.; Rasmuson, M. J.; Emrick, T.; Klier, J.; Peyton, S. R. Strain-stiffening gels based on latent crosslinking. Soft Matter 2017, 13, 9007-9014.

(13) Rosales, A. M.; Anseth, K. S. The design of reversible hydrogels to capture extracellular matrix dynamics. Nat. Rev. Mater. 2016, 1, 115.

(14) Chaudhuri, O.; Gu, L.; Klumpers, D.; Darnell, M.; Bencherif, S. A.; Weaver, J. C.; Huebsch, N.; Lee, H.-p.; Lippens, E.; Duda, G. N.; Mooney, D. J. Hydrogels with tunable stress relaxation regulate stem cell fate and activity. Nat. Mater. 2016, 15, 326-334.

(15) Nam, S.; Hu, K. H.; Butte, M. J.; Chaudhuri, O. Strainenhanced stress relaxation impacts nonlinear elasticity in collagen gels. Proc. Natl. Acad. Sci. U. S. A. 2016, 113, 5492-5497.

(16) Huang, G.; Li, F.; Zhao, X.; Ma, Y.; Li, Y.; Lin, M.; Jin, G.; Lu, T. J.; Genin, G. M.; Xu, F. Functional and Biomimetic Materials for Engineering of the Three-Dimensional Cell Microenvironment. Chem. Rev. 2017, 117, 12764-12850.

(17) Ravi, M.; Paramesh, V.; Kaviya, S. R.; Anuradha, E.; Solomon, F. D. P. 3D Cell Culture Systems: Advantages and Applications. J. Cell. Physiol. 2015, 230, 16-26.

(18) Caliari, S. R.; Burdick, J. A. A practical guide to hydrogels for cell culture. Nat. Methods 2016, 13, 405-414.

(19) Engler, A. J.; Sen, S.; Sweeney, H. L.; Discher, D. E. Matrix elasticity directs stem cell lineage specification. Cell 2006, 126, 677689.

(20) Trappmann, B.; Gautrot, J. E.; Connelly, J. T.; Strange, D. G.; Li, Y.; Oyen, M. L.; Cohen Stuart, M. A.; Boehm, H.; Li, B.; Vogel, V.; Spatz, J. P.; Watt, F. M.; Huck, W. T. Extracellular-matrix tethering regulates stem-cell fate. Nat. Mater. 2012, 11, 642-649.

(21) Kouwer, P. H. J.; Koepf, M.; Le Sage, V. A. A.; Jaspers, M.; Van Buul, A. M.; Eksteen-Akeroyd, Z. H.; Woltinge, T.; Schwartz, E.; Kitto, H. J.; Hoogenboom, R.; Picken, S. J.; Nolte, R. J. M.; Mendes, E.; Rowan, A. E. Responsive biomimetic networks from polyisocyanopeptide hydrogels. Nature 2013, 493, 651-655.

(22) Jaspers, M.; Dennison, M.; Mabesoone, M. F. J.; MacKintosh, F. C.; Rowan, A. E.; Kouwer, P. H. J. Ultra-responsive soft matter from strain-stiffening hydrogels. Nat. Commun. 2014, 5, 5808.

(23) Jaspers, M.; Rowan, A. E.; Kouwer, P. H. J. Tuning Hydrogel Mechanics Using the Hofmeister Effect. Adv. Funct. Mater. 2015, 25, 6503-6510.

(24) Jaspers, M.; Pape, A. C. H.; Voets, I. K.; Rowan, A. E.; Portale, G.; Kouwer, P. H. J. Bundle Formation in Biomimetic Hydrogels. Biomacromolecules 2016, 17, 2642-2649.

(25) Yuan, H.; Xu, J.; van Dam, E. P.; Giubertoni, G.; Rezus, Y. L. A.; Hammink, R.; Bakker, H. J.; Zhan, Y.; Rowan, A. E.; Xing, C.; 
Kouwer, P. H. J. Strategies To Increase the Thermal Stability of Truly Biomimetic Hydrogels: Combining Hydrophobicity and Directed Hydrogen Bonding. Macromolecules 2017, 50, 9058-9065.

(26) Kouwer, P. H. J.; de Almeida, P.; ven den Boomen, O.; Eksteen-Akeroyd, Z. H.; Hammink, R.; Jaspers, M.; Kragt, S.; Mabesoone, M. F. J.; Nolte, R. J. M.; Rowan, A. E.; Rutten, M. G. T. A.; Le Sage, V. A. A.; Schoenmakers, D. C.; Xing, C.; Xu, J. Controlling the gelation temperature of biomimetic polyisocyanides. Chin. Chem. Lett. 2018, 29, 281-284.

(27) Bruekers, S. M.; Jaspers, M.; Hendriks, J. M.; Kurniawan, N. A.; Koenderink, G. H.; Kouwer, P. H.; Rowan, A. E.; W, T. S. H. Fibrinfiber architecture influences cell spreading and differentiation. Cell Adh. Migr. 2016, 10, 495-504.

(28) Zimoch, J.; Padial, J. S.; Klar, A. S.; Vallmajo-Martin, Q.; Meuli, M.; Biedermann, T.; Wilson, C. J.; Rowan, A.; Reichmann, E. Polyisocyanopeptide hydrogels: A novel thermo-responsive hydrogel supporting pre-vascularization and the development of organotypic structures. Acta Biomater. 2018, 70, 129-139.

(29) Schoenmakers, D. C.; Rowan, A. E.; Kouwer, P. H. J. Crosslinking of fibrous hydrogels. Nat. Commun. 2018, 9, 2172.

(30) Van Beijnen, A.; Nolte, R.; Drenth, W.; Hezemans, A.; Van de Coolwijk, P. Helical configuration of poly(iminomethylenes). Screw sense of polymers derived from optically active alkyl isocyanides. Macromolecules 1980, 13, 1386-1391.

(31) Debets, M. F.; van Berkel, S. S.; Dommerholt, J.; Dirks, A. J.; Rutjes, F. P. J. T.; van Delft, F. L. Bioconjugation with Strained Alkenes and Alkynes. Acc. Chem. Res. 2011, 44, 805-815.

(32) Jansen, K. A.; Bacabac, R. G.; Piechocka, I. K.; Koenderink, G. $\mathrm{H}$. Cells actively stiffen fibrin networks by generating contractile stress. Biophys. J. 2013, 105, 2240-2251.

(33) Lam, J.; Truong, N. F.; Segura, T. Design of cell-matrix interactions in hyaluronic acid hydrogel scaffolds. Acta Biomater. 2014, 10, 1571-1580.

(34) Sridhar, B. V.; Brock, J. L.; Silver, J. S.; Leight, J. L.; Randolph, M. A.; Anseth, K. S. Development of a cellularly degradable PEG hydrogel to promote articular cartilage extracellular matrix deposition. Adv. Healthcare Mater. 2015, 4, 702-713.

(35) Gjorevski, N.; Sachs, N.; Manfrin, A.; Giger, S.; Bragina, M. E.; Ordonez-Moran, P.; Clevers, H.; Lutolf, M. P. Designer matrices for intestinal stem cell and organoid culture. Nature 2016, 539, 560-564.

(36) Kasza, K. E.; Broedersz, C. P.; Koenderink, G. H.; Lin, Y. C.; Messner, W.; Millman, E. A.; Nakamura, F.; Stossel, T. P.; Mackintosh, F. C.; Weitz, D. A. Actin filament length tunes elasticity of flexibly cross-linked actin networks. Biophys. J. 2010, 99, 10911100.

(37) Achilli, T. M.; Meyer, J.; Morgan, J. R. Advances in the formation, use and understanding of multi-cellular spheroids. Expert Opin. Biol. Ther. 2012, 12, 1347-1360.

(38) Iskratsch, T.; Wolfenson, H.; Sheetz, M. P. Appreciating force and shape - the rise of mechanotransduction in cell biology. Nat. Rev. Mol. Cell Biol. 2014, 15, 825-833.

(39) Huang, J. H.; Grater, S. V.; Corbellinl, F.; Rinck, S.; Bock, E.; Kemkemer, R.; Kessler, H.; Ding, J. D.; Spatz, J. P. Impact of Order and Disorder in RGD Nanopatterns on Cell Adhesion. Nano Lett. 2009, 9, 1111-1116.

(40) Yu, C. H.; Law, J. B.; Suryana, M.; Low, H. Y.; Sheetz, M. P. Early integrin binding to Arg-Gly-Asp peptide activates actin polymerization and contractile movement that stimulates outward translocation. Proc. Natl. Acad. Sci. U. S. A. 2011, 108, 20585-20590.

(41) Redick, S. D.; Settles, D. L.; Briscoe, G.; Erickson, H. P. Defining fibronectin's cell adhesion synergy site by site-directed mutagenesis. J. Cell Biol. 2000, 149, 521-527.

(42) Lam, J.; Segura, T. The modulation of MSC integrin expression by RGD presentation. Biomaterials 2013, 34, 3938-3947.

(43) Vogel, V. Unraveling the Mechanobiology of Extracellular Matrix. Annu. Rev. Physiol. 2018, 80, 353-387.

(44) Wang, H.; Abhilash, A. S.; Chen, C. S.; Wells, R. G.; Shenoy, V. B. Long-range force transmission in fibrous matrices enabled by tension-driven alignment of fibers. Biophys. J. 2014, 107, 2592-2603.
(45) Kang, H.; Wen, Q.; Janmey, P. A.; Tang, J. X.; Conti, E.; MacKintosh, F. C. Nonlinear Elasticity of Stiff Filament Networks: Strain Stiffening, Negative Normal Stress, and Filament Alignment in Fibrin Gels. J. Phys. Chem. B 2009, 113, 3799-3805. 